\title{
Serological profile of rheumatoid arthritis in a tertiary care hospital
}

\author{
P.K Surendran ${ }^{1}$ \\ ${ }^{\mathbf{1}}$ Assistant Professor, P.K Das Institute of Medical Science, Vaniyamkularn, Kerala, India
}

*Corresponding Author:

Email: surendranpkdr@gmail.com

\begin{abstract}
Rheumatoid Arthritis (RA) is an autoimmune based inflammatory pathology with involvement of joints that show early morning stiffness alongwith pain resulting in loss of function. It usually affects females aged 30 years or above. A total of 150 patients of Rheumatoid Arthritis were included in the present study of which 120 were females and 30 males (F:M=4:1). Serological tests were carried out in these patients. These test were analyzed for their sensitivity as well as specificity. Combination of various test were also assessed for their results. In the present, study age and sex of patient affected by Rheumatoid arthritis was analysed. Combination of serological tests provided a high specificity as can be seen from the present study. Quantitative Rheumatoid factor estimation and Anti Rheumatoidantibodies assay gave the maximum specificity of $89.8 \%$. A combination of serological tests is advisable rather than a single diagnostic test.This allows for the early diagnosis of Rheumatoid Arthritis so as to prevent complications by the disease.
\end{abstract}

Keywords: Investigations, Rheumatoid Arthritis, Serology, Specificity, Sensitivity.

\section{Introduction}

Rheumatoid arthritis (RA) has a prevalence of about $0.5 \%$ to $1 \%$ and an incidence of about 30 per 100 000 inhabitants, making it one of the commonest chronic inflammatory autoimmune disease. ${ }^{1-3}$ Rheumatoid arthritis (RA) is an inflammatory autoimmune pathology involving joints mainly leading to pain, early morning stiffness and restriction of movements. Cartilages, synovium, and certain systems of the body are affected with autoimmunity and inflammatory processes of RA. Early diagnosis and aggressive treatment are the best means of avoiding joint destruction, damage to organs and disability. Studies on the spectrum and expression of RA in Asian population are limited in the literature. ${ }^{5-7}$

This prospective study was carried out to determine the relationship between RA and laboratory tests used in the diagnosis of RA.Traditionally females are affected about three to four times more often than men.

\section{Materials and Methods}

This prospective study was carried out over a period of one year in a tertiary care teaching hospital between January 2017 and Dec 2017. All patients who were clinicallysuspected to be suffering from RA were included in the present study. Approval of the
Institutional Ethical committee was taken.Written consent was taken from all the patients who were included in the study. A total of 150 patients with 30 males and 120 females were included with a M:F ratio of 1:4. The age ranged from 23 years to 74 years. Patients undergoing any treatment or previously diagnosed cases of RA were excluded from the study. All patients included in the study were subjected to all the investigations such as complete haemogram, ESR, Quantitative C-Reactive protein (CRP), Rheumatoid Factor (Latex as well as quantitative), and Anti CCP Antibodies (Anti-cyclic citrullinated peptide). ESR was estimated using automated analyzer. CRP and RA factor latex test was carried out using kits from Tulip diagnostics. Quantitative assays were performed using automated analysers as per the manufacturer's instructions and protocol.

Statistical analysis was done using SPSS (Statistical Package for the Social Sciences) version 17.0 for Windows software. $p$ value of $<0.05$ was considered as statistically significant.

\section{Results}

A total of 150 cases were included in the present study. The age and sex distribution of the cases is shown in Table 1.

Table 1: Age and sex distribution of patients with rheumatoid arthritis

\begin{tabular}{|c|c|c|c|c|}
\hline S. No. & Age group & Males & Females & Total n(\%) \\
\hline 1 & $20-29$ & 3 & 28 & $31(20.7 \%)$ \\
\hline 2 & $30-39$ & 8 & 34 & $42(28.0 \%)$ \\
\hline 3 & $40-49$ & 6 & 29 & $35(23.3 \%)$ \\
\hline 4 & $50-59$ & 6 & 15 & $21(14.0 \%)$ \\
\hline 5 & $60-69$ & 4 & 12 & $16(10.7 \%)$ \\
\hline 6 & $70-79$ & 3 & 2 & $5(3.3 \%)$ \\
\hline & Total & 30 & 120 & $150(100 \%)$ \\
\hline
\end{tabular}


The male: female ratio was $1: 4$. Majority of the cases were in the age group of 30-39 years, followed closely by $40-49$ years. The mean age was 45.2 years.
Results of the Serological tests carried out in the patient are depicted in Table 2.

Table 2: Distribution of patients as per the results of the serological tests $(n=150)$

\begin{tabular}{|c|c|c|c|}
\hline S. No. & & $\mathbf{n}$ & $\mathbf{\%}$ \\
\hline 1 & Raised ESR & 126 & 84.0 \\
\hline 2 & Increased CRP & 132 & 88.0 \\
\hline 3 & Anti CCP Positive & 112 & 74.7 \\
\hline 4 & RA factor Positive & 139 & 92.7 \\
\hline 5 & High Quantitative RA factor & 143 & 95.3 \\
\hline
\end{tabular}

Quantitative RA factor was raised in 143 of the 150 patients (95.3\%). Anti CCP antibodies were present in only 112 of the 150 patients $(74.7 \%)$.There has been an observed overlap between the serological tests also. In some patients multiple parameters were positive or their values were more than the normal. We also studied the sensitivity and specificity of different serological markers as well as their combinations (Table 3).

Table 3: Sensitivity and Specificity of different serological tests in the present study

\begin{tabular}{|l|c|c|}
\hline & Sensitivity & Specificity \\
\hline ESR & $92.1 \%$ & $68.2 \%$ \\
\hline CRP & $84.1 \%$ & $70.4 \%$ \\
\hline Anti CCP Antibodies & $74.2 \%$ & $88.5 \%$ \\
\hline R A factor (Latex) & $38.2 \%$ & $87.8 \%$ \\
\hline Quantitative R A factor & $71.8 \%$ & $64.6 \%$ \\
\hline Anti CCP + Q R A & $71.4 \%$ & $94.1 \%$ \\
\hline Anti CCP + Latex R A & $52.4 \%$ & $89.8 \%$ \\
\hline
\end{tabular}

Combination of test increases the specificity rates significantly. It was further observed that patients with multi joint involvement if tested negative for both latex RA factor as well as Anti CCP antibodies indicate that the patient is not having rheumatoid arthritis. There was a positive correlation between anti-CCP antibodies and higher ESR, CRP. Also there was a positive correlation between RF and increased ESR and CRP.In this study fifty percent of the patients were both anti-CCP and RF positive. The study $(\mathrm{p}<0.05)$ revealed significant positive correlation between RF and anti-CCP antibodies.

\section{Discussion}

Rheumatoid Arthritis is a systemic disease, accompanied by progressive joint destruction and deformity. Depending on the severity, there may also be extra-articular manifestations, involving skin, vascular channels, and internal organs as well. If inadequately treated, RA leads in the long term to a significant impairment of the quality of life; morbidity and increase in mortality. Early diagnosis and suitable therapy are therefore of great importance in determining the prognosis of RA. The three pillars for the diagnosis of rheumatological disease are a good medical history, clinical findings (including radiological investigations) and serological laboratory tests.

Serological diagnostic testing is of growing importance in the early detection and differentiation of rheumatoid arthritis. Apart from the traditional detection of rheumatoid factor, new specific autoantibodies to citrullinated antigens have made a crucial contribution to the diagnosis of RA. The rheumatoid factor is an autoantibody, which may be $\mathrm{IgM}, \operatorname{IgG}$ or $\operatorname{IgA}$, and which was first mentioned in $1922 .{ }^{1}$ It recognizes domains $\mathrm{CH} 2$ and $\mathrm{CH} 3$ of the $\mathrm{Fc}$ segment of human IgG and is a component of the classification criteria for RA published by the American College of Rheumatology. ${ }^{2}$

The classification criterion to define RA that is used internationally was defined by the American College of Rheumatology (ACR) in 1987. New criteria for rheumatoid arthritis classification were introduced in $2010 .{ }^{1}$ RA sera contained antibodies that reacted to the keratinized layer of epithelial cells. These were called antikeratin antibodies, and were reported only in RA patients. Anti-CCP was included in the ACR/EULAR (European League against Rheumatism) RA classification criteria in 2010. Erythrocyte sedimentation rate (ESR), C-reactive protein (CRP), and rheumatoid factor (RF) are the other serological tests used in the RA classification criteria. Assessment of anti-CCP, RF, ESR and CRP in the serum was included in the ACR/EULAR RA classification criteria in 2010. Several studies have questioned the importance of anti-CCP antibody testing in distinguishing RA from other inflammatory diseases. ${ }^{1-2}$ Joints that showed erosion or destruction showed strong association with anti CCP antibodies. ${ }^{3}$ RA has been associated with several autoantibodies, including rheumatoid factors (RF), anti-perinuclear factor (APF), anti-keratin antibodies (AKA) and anti-filaggrin antibodies (AFA) ${ }^{4}$ 
These autoantibodies bind antigenic determinants that contain the unusual amino acid citrulline, formed by a post-transcriptional modification of arginine residues by peptidylarginine deiminase. To detect these autoantibodiesELISA is used. This method uses as antigen a cyclic variant of a citrullinated peptide (CCP) derived from the sequence of human filaggrin. The anti-CCP antibody test is now commercially available and its diagnostic accuracy is comparable with that of the RF test ${ }^{5}$. The diagnostic accuracy of this test in the detection of RA is still unclear, as sensitivities ranging from 41 to $68 \%$ have been reported. ${ }^{3,6,7}$ In the present study a comparison of the sensitivity and specificity of the anti-CCP antibody test with those of other tests commonly used in the diagnosis of RA. Role of these autoantibodies in assessing the severity of the disease is unknown. ${ }^{8-10}$ The present study establishes the value of anti-CCP antibodies in the diagnosis of RA both singly as well as in combination. The specificity obtained for the anti-CCP test $(88.5 \%)$ was almost similar to that found by other groups: 91-98\%, ${ }^{1,8-10}$ However, although there is consensus by different authors regarding specificity, there is significant variation in the sensitivity, rates ranging from $41-68 \%$. This variation may be attributed to the different dilutions of serum used or, to the different cut-off values as per the diagnostic test employed.

The anti-CCP antibody test has moderate sensitivity and excellent specificity, the AKA test has poor sensitivity but excellent specificity, and the IgM RF test has moderate sensitivity and specificity ${ }^{11-15}$. A positive test for anti-CCP antibodies or AKA practically establishes this diagnosis. Hence these tests, may prove more useful in selected cases in clinical practice especially in cases where significant disagreement between these tests exist, True positives among RA patients and false positives among controls, suggests that there is considerable room for improvement in the serological diagnosis of RA. ${ }^{16}$ Whether these tests are able to predict the occurrence of clinical or radiological manifestations of RA remains a mystery. The present study was cross-sectional and therefore liable to possible selection bias. Prospective studies would probably have produced more information than a cross-sectional study of patients with definite RA. However, in prospective studies, it is mandatory to predict that treatments have not affected the results. ${ }^{17}$

\section{Conclusion}

In conclusion, our results suggest that anti-CCP antibodies, AKA and IgM RF reflect clinically relevant disease processes in RA patients. However, in clinical practice, both IgM RF and anti-CCP antibodies may be useful, IgM RF for their good sensitivity and as a marker of disease severity and anti-CCP antibodies for their high specificity and their presence in some RA-seronegative patients.

\section{References}

1. Tennakoon TMIS, Nissanka TM, Bandaranayake BMVC. The spectrum of Rheumatoid Arthritis. A single unit experience. Anuradhapura Medical Journal. 2015;9(2Supp):S38.

2. Aletaha D, Neogi T, Silman AJ, Funovits J, Felson DT, Bingham CO, et al. An American College of Rheumatology/ European League Against Rheumatism collaborative initiative 2010 rheumatoid arthritis classification criteria. Arthritis Rheum. 2010;62:25692581.

3. Visser H, le Cessie S, Vos K, Breedveld FC, Hazes JMW. How to diagnose rheumatoid arthritis early: A prediction model for persistent (erosive) arthritis. Arthritis Rheum. 2002;46:357-365.

4. Goldbach-Mansky R, Lee J, McCoy A. Rheumatoid arthritis associated autoantibodies in patients with synovitis of recent onset. Arthritis Res. 2000;2:236-43.

5. Schellekens GA, Visser H, de Jong BA. The diagnostic properties of rheumatoid arthritis antibodies recognizing a cyclic citrullinated peptide. Arthritis Rheum. 2000;43:155-63.

6. Bizzaro N, Mazzanti G, Tonutti E, Villalta D, Tozzoli R. Diagnostic accuracy of the anti-citrulline antibody assay for rheumatoid arthritis. Clin Chem. 2001;47:1089-93.

7. Gupta R, Thabah MM, Aneja R, Chandrasenan PJ. Usefulness of Anti-CCP antibodies in rheumatic diseases in Indian patients. Indian J Med Sci. 2009;3:92.

8. Kashyap B, Tiwari U, Garg A, Kaur IR. Diagnostic utility of anti-CCP antibodies and rheumatoid factor as inflammatory biomarkers in comparison with C-reactive protein and TNF-Y in rheumatoid arthritis. Trop J Med Res. 2015;18:5-9.

9. Lee DM, Schur PH. Clinical utility of the anti-CCP assay in patients with rheumatic disease. Ann Rheum Dis. 2003;62:870-874.

10. Swedler W, Wallman J, Froelich CJ, Teodorescu M. Routine measurement of $\operatorname{IgM}, \operatorname{IgG}$ and IgA rheumatoid factors: High sensitivity, specificity and predictive value for rheumatoid arthritis. J Rheumatol. 1997;6:1037-44.

11. Arnett FC, Edworthy SM, Bloch DA. The American Rheumatism Association 1987 revised criteria for the classification of rheumatoid arthritis. Arthritis Rheum. 1988;31:315-24

12. Schellekens GA, Visser H, de Jong BA. The diagnostic properties of rheumatoid arthritis antibodies recognizing a cyclic citrullinated peptide. Arthritis Rheum. 2000;43:155-63.

13. Ahmad A, Singh TB, Usha. An epidemiological study on clinical suspected rheumatoid arthritis rural patients of Eastern Uttar Pradesh, India. IRMJCR. 2014;2(1):48-54.

14. Bharathi KV, Sobharani YN, Sridhar C. Epidemiological study on rheumatoid arthritis. Indian Journal of Multidisciplinary Research. 2009;5(1):17-24.

15. Malaviya A, Kapoor S, Singh R, Kumar A, Pande I. Prevalence of rheumatoid arthritis in the adult Indian population. Rheumatol Int. 1993;13:131-4.

16. Ropes MW, Bennett GA, Cobb S, Jacox R, Jessar RA. Revision of diagnostic criteria for rheumatoid arthritis Bull Rheum Dis. 1958;9:175.

17. Vliet Vlieland TP, Buitenhuis NA, van Zeben D, Vandenbroucke JP, Breedveld FC, Hazes JM. Sociodemographic factors and the outcome of rheumatoid arthritis in young women. Ann Rheum Dis. 1994;53(12):803-6. 